\title{
Im Schraubstock der Angst: Manipulation und unsere Disposition zur Ängstlichkeit
}

\section{Alexander Fischer ${ }^{1}$}

Angst scheint einer der größten Motivatoren im Leben menschlicher und nicht-menschlicher Tiere zu sein. So ist sie einerseits ein grundlegender Automatismus, wenn wir von der Angst als wirksames evolutionär entstandenes Schutzsystem sprechen, das nahe und ferne Gefahren erkennt und in der Folge eine der Schutzreaktionen Angreifen, Flüchten oder Einfrieren auslöst. ${ }^{2}$ Andererseits betrifft sie im Verständnis verschiedener Existenzphilosophien die Seinsbestimmung unseres Daseins, gilt mit dem dänischen Philosophen Sören Kierkegaard als die andere Seite der Medaille der Freiheit ${ }^{3}$ und ist auch so ein Grundphänomen der menschlichen Existenz. Schliesslich müssen wir freiheitlich entscheidend handeln, können uns dabei aber nicht immer sicher sein, ob wir denn richtig agieren. Das Pendeln zwischen Sicherheit und Angst gehört zu unseren grundsätzlichen und bestimmenden Lebensbewegungen. Angst, ich mache in diesem Aufsatz keinen Unterschied zur »Furcht $\aleph^{4}$, kann dabei unter-

1 Mein Dank gilt Eleonora Meister, Yvonne Hohnhausen und Markus Höfner für die gründliche Lektüre und die hilfreichen Anmerkungen.

2 Sven Olaf Hoffmann, Der verborgene Sinn (Nutzen) menschlicher Ängste, in: Jahrbuch für Literatur und Psychoanalyse 38 (2019), 177-190, hier 182ff.

${ }_{3}$ Für Martin Heidegger wiederum eröffnet sich in der Angst die Endlichkeit und Nichtigkeit unserer Existenz. Angst wirft uns also auch hier auf unser Dasein zurück. Andere Existenzphilosophen binden das Phänomen jeweils auf ihre Art ein. Genauer kann in diesem Artikel darauf nicht eigegangen werden.

4 Ich möchte kurz auf die klassische Unterscheidung von Sören Kierkegaard (und später auch Heidegger) zwischen "Furcht" als intentional auf einen konkreten Gegenstand gerichteter Empfindung und "Angst« als einer auf das Selbst gerichteten, auf das Ganze gehenden Empfindung reagieren (Vgl. Sören Kierkegaard, Der Begriff Angst. Eine schlichte psychologisch-andeutende Überlegung in Richtung auf das dogmatische Problem der Erbsünde, von Vigilius Haufniensis, in: Gesammelte Werke in 36 Abteilungen, übersetzt und hg. v. Emanuel Hirsch/Hayo Gerdes, Gütersloh 1979-1986, Abt. 11, 40). Mir ist diese Unterscheidung nicht notwendig einleuchtend: Sowohl Angst als auch Furcht sind reflexiv auf das eigene Selbst bezogen, dem es um das eigene Wohl, Leben oder Sein geht, sie antizipieren eine mehr oder weniger klare Bedrohung im Außen (z.B. eine echte Spinne auf der einen, aber auch etwas mittels der Phantasie Imaginiertes auf der anderen Seite) und enthalten dabei Motivationen in Richtung Angriff, Flucht oder Einfrieren. Ich verstehe Angst daher als graduellen Begriff, der eben mehr oder weniger objektgerichtet sein kann (Vgl. auch Chris- 
schiedlichste, oft individuell ausgeprägte, Gestalten annehmen und konkret und gegenstandsbezogen oder diffus und alles überlagernd sein. Sie kann auch unterschiedlichste Intensitäten annehmen, indem sie körperlich manifestiert von leichter, unterschwelliger Ängstlichkeit bis zu handfester, unkontrollierbarer Todesangst variiert; damit einher gehen verschiedene Grade an kognitiver und körperlicher Belastung. ${ }^{5}$ Auch die Gegenstände der Angst variieren stark; sie mag auf eine konkrete körperliche Bedrohung, das Scheitern eines Plans, einen öffentlichen Auftritt, oder die bloße Imagination irgendeiner Bedrohung - was dann wiederum krankhaft ausgeformt sein kann - bezogen sein. Gerade der imaginative Charakter von Angst spielt eine grosse Rolle in ihrer Ausprägung. ${ }^{6}$ Diese Vielschichtigkeit bedeutet auch: "Zwischen Angst als elementarem Leibzustand der Beklemmung, als intentional gerichteter Emotion und als Stimmung der Unheimlichkeit lassen sich dabei keine scharfen Grenzen ziehen; die verschiedenen Formen gehen häufig ineinander über. « ${ }^{7}$

Die grundsätzliche Gegenwärtigkeit als Automatismus, Emotion und Existenzial sowie die Vielfältigkeit der Ausformung machen Angst zu einer wesentlichen Disposition unseres Handelns. Sie ist so ein Regulativ, das wir nicht selten in uns am Werke spüren können und das ein in der Interaktion mit der Umwelt allgemein wirksamer Faktor ist - sei es bezogen auf Gegenständliches oder Personen. Indem Angst konkret und gegenstandsbezogen geschürt, in ihrer mitunter diffusen Beschaffenheit angestoßen wird, oder aber als unterliegende Folie in primär auf andere Emotionen - wie Schuld und Scham oder auch Eifersucht - bezogenen Entscheidungskontexten fungieren kann, lässt sie sich gezielt zur Beeinflussung der Handlungsweisen von Akteuren nutzen. So eröffnet sich ein manipulatives Potential der Angst. Wir kennen das aus unserem Alltag,

toph Demmerling/Hilge Landweer, Philosophie der Gefühle.Von Achtung bis Zorn, Stuttgart 2007, 80ff.).

5 Angst ist kein rein kognitives Problem, je länger sie anhält, desto deutlicher manifestiert sie sich auch in langfristig sichtbaren körperlichen Reaktionen.

6 Sie bedingt auch eine ganz spezielle Form der Angst: die Angst vor der Angst. Also die Angst vor dem Ausgeliefertsein all der körperlichen und kognitiven Folgen von Angsterleben. Anders gesagt: die Angst vor Kontrollverlust.

7 Thomas Fuchs/Stefano Micali, Die Enge des Lebens. Zur Phänomenologie und Typologie der Angst, in: Angst. Philosophische, psychopathologische und psychoanalytische Zugänge, hg. v. Stefano Micali/Thomas Fuchs, Freiburg i.Br./München 2017, 98-118, hier 98. Ich bin nicht sicher, warum der elementare Leibeszustand von den anderen beiden Grossbereichen der Angst abgegrenzt ist, da diese ja auch mit konkreten leiblichen Zuständen einhergehen. Auch muss die Stimmung nicht zwingend unheimlich sein. Nichtsdestotrotz gehe ich d'accord mit der Anmerkung, dass eine klare Abgrenzung der Angstphänomene voneinander schwierig ist. 
aus Beziehungen mit anderen Menschen, aber auch aus politischen Kontexten. Es ist dieser interpersonale Wirkungsraum der Angst, auf den ich ein Schlaglicht werfen möchte.

Im Folgenden soll also die Funktionsweise von Angst im Rahmen der Manipulation aufgeschlüsselt werden. "Manipulation« verstehe ich dabei als eine Form der Beeinflussung, die primär auf der affektiven Ebene operiert. Wenngleich ich davon ausgehe, dass unsere rationalen Fähigkeiten und unsere affektive Beschaffenheit nicht als Dichotomie zu denken sind, sondern in einem steten Wechselspiel stehen. Die Abtrennung "affektiv« vs. "rational« ist also idealtypisch. Damit liegt die Manipulation zwischen dem rationalen Überzeugen mit guten Gründen auf der einen und der (oft gewaltsamen) Entscheidungseinbahnstrasse Zwang auf der anderen Seite innerhalb eines Kontinuums der Beeinflussung. Wenn wir nun von Manipulation als Beeinflussungsform auf affektiver Ebene sprechen, agiert sie im Dreischritt: Als erstes wird eine Handlungsoption gezielt mit einer angenehmen oder - hierher gehört die Angst - unangenehmen Empfindung verbunden. ${ }^{8}$ Somit erscheint es uns attraktiver oder unattraktiver, auf eine bestimmte Art und Weise zu handeln. Dementsprechend wird es auch wahrscheinlicher oder unwahrscheinlicher, dass wir diese Handlung tatsächlich ausführen. Der Manipulator legt hier also die Wahl eines Zweckes nahe, zwingt aber nicht zur Wahl. Der Manipulierte bleibt frei, diesen Zweck zu seinem zu machen - oder nicht. ${ }^{9}$ Angst eignet sich hierfür direkt und indirekt in ihrer Wesenheit als allgemeine Basisemotion ${ }^{10}$, die einem Schraubstock ähnlich - wie Stefan Zweig sie in seiner Novel-

\footnotetext{
8 Lustvolle Angst wie jene, die wir gezielt z.B. im Schauen eines Horrorfilms oder Lesen eines blutrünstigen Krimis induzieren, lasse ich hier aussen vor und fokussiere somit auf den generell unangenehmen Aspekt der Angst.

9 Vgl. Alexander Fischer, Manipulation. Zur Theorie und Ethik einer Form der Beeinflussung, Berlin 2017, Alexander Fischer/Christian Illies, Modulated Feelings. The Pleasurable-Ends-Model of Manipulation, in: Philosophical Inquiries IV/2 (2018), 25-44 sowie auch Alexander Fischer, Ein Parasit im Kokon des Schmetterlings? Manipulation, Kommunikation und Ethik, in: Klaus Sachs-Hombach/Bernd Zywietz, Fake News, Hashtags \& Social Bots. Neue Methoden populistischer Propaganda, Wiesbaden 2018,14-49. Manipulation ist ein umstrittenes Konzept. Dies hier ist ein Ansatz Manipulation zu konzeptualisieren. Es hat weitere Versuche gegeben, auf die ich im entsprechenden Unterkapitel kurz verweisen werde. Im Buch »Manipulation" findet sich eine kritische Auseinandersetzung mit diesen Ansätzen.

${ }^{10}$ Angst scheinen wir tatsächlich kulturübergreifend feststellen zu können, wenngleich ihr spezielles Ablaufmuster durchaus unterschiedlich und von kulturellen Faktoren eingefärbt sein kann. Ich beziehe mich hier vornehmlich auf den Erfahrungsraum der Angst in westlichen Gesellschaften.
} 
le Angst treffend bezeichnet ${ }^{11}$ - bestimmte Entscheidungsmomente fester oder weniger fest im Griff haben kann. Bevor wir aber ihr manipulatives Potential ausleuchten, versuchen wir zuerst, etwas mehr Licht ins Dickicht der komplexen und vielfältigen Wesenheit von Angst zu bekommen. Denn Angst ist nicht gleich Angst, wie wir schon oben gesehen haben. Sigmund Freud meinte, sie sei eine "allgemein gangbare Münze « ${ }^{2}$ im seelischen Haushalt und stecke »[i] rgendwie [...] hinter allen Symptomen $«{ }^{13}$ Er nahm zunächst an, dass eigentlich alle Affekte durch Verdrängung derart veränderbar sind, dass sie sich als Angst äussern, wenn die verdrängten Affekte einen Weg nach Aussen suchen. Später revidierte er dieses Konzept (in seiner Vorlesung "Angst und Triebleben") dahingehend, dass Angst nicht mehr nur das Ergebnis von Verdrängung sein kann, sondern gar ihre Grundlage: Die Angst mache mitunter die Verdrängung und steckt so auch hinter dieser Symptomatik. Interessant ist, zu fragen, ob es etwas allen Angsterfahrungen Verbindendes gibt. Im Auftrag grösserer Klarheit möchte ich mit diesem Ziel Angst kurz mit Hilfe der Literatur phänomenologisch vergegenwärtigen und ihre wesentlichen Charakteristika herausarbeiten. Sodann soll das bereits angerissene Verständnis der Manipulation vertieft und mit Angst verknüpft werden, um besser zu verstehen, in welchen hauptsächlichen Formen Angst manipulativ genutzt werden kann. Im abschließenden Ausblick werde ich kurz die ethische Problemstellung des manipulativen Einsatzes von Angst thematisieren.

\section{Vergegenwärtigung der Angst}

In Anbetracht der Vielschichtigkeit und auch Individualität der Angst und ihrem besonderen Status als Kehrseite aller anderen Affekte nach Freud, hilft es also zu fragen, ob etwas alle Ausprägungen der Angst miteinander verbindet. Nützlich ist deshalb, eine gezielte Phänomenologie der Angst zu betreiben, also ihre allgemeinen Charakteristika zu ermitteln. Die Weltliteratur hält hierfür treffende Vergegenwärtigungen bereit. Beginnen wir mit einer gerafften Darstellung eines Angstzustandes, den Franz Kafkas Josef K. im Roman Der Proceß durchmacht, nachdem er verraten wurde und zum ersten

\footnotetext{
${ }^{11}$ Stefan Zweig, Angst. Novelle, Stuttgart 2013, 11.

${ }^{12}$ Sigmund Freud,Vorlesungen zur Einführung in die Psychoanalyse, in: Gesammelte Werke, Bd. 11, Frankfurt a.M. 1969, 390.

${ }^{13}$ Sigmund Freud, Das Unbehagen in der Kultur, in: Gesammelte Werke. Chronologisch geordnet, Bd. 14, London 1955, 495.
} 
Mal auf den seltsam anmutenden Dachböden der Kanzleien unterwegs ist:

"Aber sein stummes Dastehn mußte auffallend sein [...]. . Wollen Sie sich nicht setzen?< K. setzte sich sofort und stützte, um noch bessern Halt zu bekommen, die Elbogen (sic!) auf die Lehnen. 'Sie haben ein wenig Schwindel, nicht? fragte sie ihn. [...] 'Machen Sie sich darüber keine Gedanken<, sagte sie, >das ist hier nichts Außergewöhnliches, fast jeder bekommt so einen Anfall, wenn er zum ersten Mal herkommt. [... < [ ...] K. antwortete nicht, es war ihm peinlich, durch diese plötzliche Schwäche den Leuten hier ausgeliefert zu sein. [...] Überdies war ihm [...] noch ein wenig schlechter, [...] die Frisur war zerstört, die Haare hingen ihm in die schweiß-bedeckte Stirn. [...] >Nein<, sagte K., rich will mich nicht ausruhn.८ Er hatte das mit möglichster Bestimmtheit gesagt, in Wirklichkeit hätte es ihm aber sehr wohlgetan sich niederzusetzen; er war wie seekrank. Er glaubte auf einem Schiff zu sein, das sich in schwerem Seegang befand. Es war ihm als stürze das Wasser gegen die Holzwände, als komme aus der Tiefe des Ganges ein Brausen her, wie von überschlagendem Wasser, als schaukle der Gang in der Quere und als würden die wartenden Parteien zu beiden Seiten gesenkt und gehoben. Desto unbegreiflicher war die Ruhe des Mädchens und des Mannes, die ihn führten. Er war ihnen ausgeliefert, ließen sie ihn los, so mußte er hinfallen wie ein Brett. [...] Endlich merkte er, daß sie zu ihm sprachen, aber er verstand sie nicht, er hörte nur den Lärm der alles erfüllte und durch den hindurch ein unveränderlicher hoher Ton wie von einer Sirene zu klingen schien. ${ }^{14}$

Der Protagonist aus Kafkas Roman durchlebt hier gleich mehrere Facetten der Angst, die sich an das annähern, was wir eine Angstoder Panikattacke nennen. Kafkas Sprache selbst vermag uns bereits in die Empfindung zu ziehen und mit ihren vielen Nebensätzen die Kaskaden der Angst zu vergegenwärtigen. Die hier dargestellten Facetten des ängstlichen Erlebens ermöglichen uns eine treffende Beschreibung davon, was im Allgemeinen passiert, wenn wir Angst haben - auch wenn nicht jedes einzelne Symptom in jedem Fall bei jeder Person auftreten muss. Dröseln wir die Dinge ein wenig auseinander.

Zunächst ist auffällig, dass K. körperlich reagiert: Er steht einfach da, matt, steif wie ein Brett und unfähig sich vorwärts oder rück-

${ }^{14}$ Franz Kafka, Der Proceß. Roman in der Fassung der Handschrift, Frankfurt a.M. 2010, 79-85. 
wärts zu bewegen. Schwindel steigt in ihm auf, er ist unsicher im Gang. Er sehnt sich danach, sich zu setzen und an den Armlehnen des Stuhls Halt zu gewinnen. Er schwitzt, ihm ist übel, er ist in Unordnung, fühlt sich schwächlich und ist zugleich doch agitiert - muss irgendwie raus aus der Situation. K. ist hochempfindlich für die Reize um ihn herum. Gleichzeitig ist er benommen, unter einem Schleier, der ihn die Gegebenheiten verzerrt wahrnehmen lässt. Das führt dazu, dass K. das Verhalten der ihn umgebenden Personen nur schwer zu deuten weiss und ambivalent auf den Ort und die Anwesenden bezogen ist. Anders gesagt: K. zeigt klassische vegetative Stresssymptome, die sich auch vor dem Hintergrund von Angst immer deutlich bemerkbar machen und die Kognition, das rationale Denken, verschleiernd affektieren. Deutlich wird, dass ein "gehindertes Weg! «, wie es der Philosoph Hermann Schmitz nennt ${ }^{15}$, die seltsam ambivalente Situation zwischen (körperlicher und auch mitunter räumlich wahrgenommener) Einengung und dem Wunsch daraus auszureissen grundiert - bei gleichzeitiger Hemmung des Ausreissimpulses, was wiederum Angst zu steigern vermag. Es zeigt sich ein für Angst typischer Kontrollverlust, der sich körperlich, aber auch kognitiv manifestiert. Resultate davon sind diffuse Bewegungen oder Starre, Vermeidung sowie steigende innere Spannungen (die auch bis zur Aggression und in eventueller Folge einem Angriff reichen können). ${ }^{16}$

Die Szene zeigt uns dabei auch, wie sehr K. durch die Angst von der Aussenwelt abgetrennt wird. ${ }^{17}$ Er kann die um ihn Sprechenden nicht mehr richtig hören, ist in schmerzlichem Bewusstsein der eigenen Leiblichkeit auf sich selbst zurückgeworfen. Dabei sind nur noch Lärm und ein hoher Ton im Kopf. Die Angst verkörpert sich also in einer Art Tinnitus. K. schämt sich zudem für seinen Zustand, die Sichtbarkeit seiner Angst und die damit einhergehende erwartete negative Bewertung, er fühlt sich machtlos und so den Menschen um ihn herum ausgeliefert. Auch Scham, die oft eine Folge eines Angstzustandes ist, trennt uns tendenziell vom positiven Kontakt mit der Aussenwelt ab, wir würden am liebsten unsichtbar werden. In anderen Worten: K.s Bewegungs- und Lösungsradius ist klein, sein Handlungsspielraum eingeschränkt. Wenn er auch eigentlich flüchten will, ist er festgesetzt. Es wird hier noch etwas deutlich: Angst grundiert oft andere Affekte wie Scham oder auch Schuld, die eine

${ }^{15}$ Hermann Schmitz, System der Philosophie, 1. Band: Die Gegenwart, Bonn 1981, $169 f$.

${ }^{16} \mathrm{Vgl}$. Fuchs/Micali, Die Enge des Lebens, $98 \mathrm{ff}$.

17 Vgl. auch ebd., 99. 
enge Verzahnung mit ihr haben, da sie sich um drohende negative Konsequenzen und den Verlust von Anerkennung als ein liebenswertes menschliches Wesen drehen.

Eine konkret-gegenständliche Bedrohung ist in der zitierten Szene dabei nicht ohne Weiteres auszumachen. Auch das kann als charakteristisch für Angst gelten, ist vielleicht sogar typischer (und jedenfalls für den Rahmen der Manipulation ungleich spannender) als die direkte Bedrohung durch einen Tiger (zumindest in unseren Breiten). K.s Angst ist eher multifaktoriell und dabei diffus (derVerrat, die Anklage, undurchschaubare Räumlichkeiten, unausrechenbare Personen vor Ort, ...). Ihm ist die "Objektivierung der Situation" erschwert, sogar weitgehend unmöglich (obwohl K. es probiert), und doch rasen die Gedanken, denn:»Angst neigt zur Illusionsbildung «. ${ }^{18}$ Sein Kopf hat Spielraum für Phantasie. Die Imagination wird hier als wesentlicher Bestandteil von Angst deutlich und wirkt kognitiv wie affektiv belastend. Man spricht mitunter auch vom »Katastrophisieren" oder - Kierkegaard hat das wunderbar treffend ausgedrückt - der »Kreativität der Selbstqual $\ll{ }^{19}$ Kafka vergegenwärtigt K.s Angst sodann auch in einer eindringlichen Phantasie: Der Gang verwandelt sich in ein Schiffsdeck, das nicht nur brutal, unkontrollierbar schaukelt und bedrohliche Wellenbrüche erleidet, sondern auch von aus der Tiefe kommender, dunkler Bedrohung - der Angstquelle - zeugt. Hier wird uns der drohende Verlust des Verstandes vor Angst anschaulich gemacht und die oben geschilderten körperlichen Symptome werden im Nachgang an den negativen Film, der im Kopf abläuft und aufwiegelnde Wirkung hat, noch nachvollziehbarer. Wiederum anders gesagt: Die Phantasie spielt K. Streiche, blüht in bedrohlichen Szenarien und das bleibt nicht folgenlos; der Schraubstock der Angst erfährt hieraus wesentlich die Festigkeit seines Zugriffs. Ein Tiger verschwindet hoffentlich irgendwann von selbst; zudem: Gegen konkrete Angstauslöser kann meist auch konkret zur Ausräumung vorgegangen werden. Die Vorstellung einer Bedrohung aber - zumal wenn sie diffus ist - lässt sich ungleich schwerer in den Griff bekommen. Und wie Walter Lippmann weiss: »For it is clear enough that under certain conditions men respond as powerfully to fictions as they do to realities. $\aleph^{20}$ Dem menschlichen Gehirn ist es gleich, ob eine Bedrohung real oder fiktiv ist (daraus ergibt sich nicht zuletzt die Möglichkeit an diffusen Ängsten im therapeutischen Kontext mit Kopf und Körper zu arbeiten). Die

\footnotetext{
${ }^{18}$ Vgl. ebd., 101 und 103.

${ }^{19}$ Kierkegaard, Der Begriff Angst, 161.

${ }^{20}$ Walter Lippmann, Public Opinion, Miami 2008, 14.
} 
intersubjektiv nachvollziehbare Komponente einer Bedrohung ist für ihre Wirkung im Ängstlichen also nicht notwendig von Belang; so hat in der Kafka-Szene auch niemand sonst ein Angsterleben (im Gegenteil: K.s Erleben wird oft durch lachende Andere kontrastiert). So kreisen die Gedanken, tendieren zu negativen Interpretationen, fürchten Bewertungen und unsere psychische Ökologie, also die Balance unseres psychischen Haushaltes - oder anders gesagt: die Möglichkeit uns selbst und unser Handeln in Verbindung mit der Umwelt unverrückt wahrzunehmen und zu verorten -, ist verzerrt und wacklig geworden. ${ }^{21}$ Es fehlt der sichere Halt in der Realität.

Neben den vielfältigen vegetativen Symptomen, die sich allgemein als Stresssymptome charakterisieren lassen, wird deutlich, dass alle Angst darin verbunden ist, dass sie eine Einschränkung des freien Handlungsspielraumes bedeutet (bis zum Kontrollverlust), die grundiert wird durch a) ein Ausreissen wollen bei gleichzeitiger Gehemmtheit dieses Impulses, b) eine Abtrennung von der Aussenwelt in manifester Ambivalenz sowie (c) phantastischer Imagination, die ein Bedrohungsszenario in kräftigen Farben ausmalt und mitunter zu vergrössern vermag. Dass Angst oft nicht viel mit der Realität zu tun hat, sondern mit einer möglichen, suggerierten Realität, machen uns solche Manipulationen deutlich, die sich Angst zunutze machen. Hierbei geht es nicht zwingend um echte Bedrohungen, sondern meist um diffuse oder gar gänzlich fingierte. Die imaginierte und dennoch deutlich gefühlte Bedrohung, die damit einhergehende (befürchtete) Einschränkung des Handlungsspielraumes und die schmerzvoll drohende Getrenntheit lassen sich in zwischenmenschlichen Beziehungen sowie im politischen Kontext vielfach nutzen. Wir können so eine erste Typologie der angstbezogenen Manipulation skizzieren: Es lässt sich (1) eine diffuse Stimmung der Angst etablieren, die sich in der angeregten Anschlussfähigkeit an die Phantasie der Einzelnen für Manipulationen eignet. Dies ist der wohl häufigste Fall der Nutzung von Angst im manipulativen Kontext. Gleichzeitig lassen sich aber auch die Abtrennung von der Aussenwelt und die Gehemmtheit des Ausreissenwollens manipulativ einspannen, indem dieses Ausgeliefertsein (2) in einer konkret in Bezug auf etwas bestehenden Angstsituation für die Erreichung der Zielsetzungen von Manipulatoren genutzt wird. (3) lassen sich angstverwandte Emotionen wie Schuld, Scham oder Eifersucht manipulativ beeinflussend nutzen, die mit ihrer engen Verbindung zur Angst ähnliche Manifestationen affektiver und kognitiver Art zeitigen. Auch dies ist eine idealtypische Aufteilung. Die drei aufgezählten Modulationsversuche vermischen sich oft und werden sowohl in Beziehungen

${ }^{21}$ Fischer, Manipulation, 165. 
als auch im Politischen flexibel genutzt, je nachdem, wie ein Ziel am besten erreichbar ist. Schauen wir uns die manipulativen Weisen und ihre Voraussetzungen im Folgenden etwas genauer an.

\section{Manipulation und das Mittel der Angst}

"Manipulation" ist ein zwiespältiger Begriff. Zwar ist er einerseits fest in unserer Sprache verankert und wird dort als klar negative Form der Beeinflussung charakterisiert. Andererseits ist man sich im wissenschaftlichen Diskurs nicht einig, wie Manipulation am sinnvollsten zu konzeptualisieren ist - und ob hierbei neutral oder normativ aufgeladen definiert werden darf. ${ }^{22}$ Alltagssprachlich wird Manipulation verstanden als Bezeichnung für eine Beeinflussungsform, die Menschen in absichtlich täuschender und egoistischer Manier zu etwas bringt, was sie nicht wollen, bzw. die durch eine verschleierte Zielsetzung bestimmt ist und nachteilig für die manipulierte Person sein wird. Sie gilt sogar als eine Art "psychischer Fesselung«, ein Zwang, so dass Menschen gar nicht mehr anders handeln könnten, als es ein Manipulator vorsieht. ${ }^{23}$ Dies ist jedoch irreführend. Nicht nur, weil Täuschung,Verschleierung und negative Konsequenzen Teil der Manipulation sein können, aber nicht notwendig sein müssen. ${ }^{24}$ Sondern auch, weil begrifflich die Grenzen $\mathrm{zu}$ anderen Formen der Beeinflussung, nämlich Täuschung und

\footnotetext{
${ }^{22}$ Siehe für einen Überblick über die Diskussion Fischer, Manipulation, Kapitel 1. Zur ersten weiteren Vertiefung und alternative Konzepte siehe den Sammelband Christian Coons/Michael Weber (Hg.), Manipulation. Theory and Practice, Oxford 2014.

${ }^{23} \mathrm{Vgl}$. für den alltagssprachlichen und nicht wissenschaftlich auseinandergesetzten Begriff von Manipulation Hellmuth Benesch/Walter Schmandt, Manipulation und wie man ihr entkommt, Stuttgart 1979, $7 \mathrm{ff}$.

${ }^{24}$ Täuschungen sind insofern nicht notwendig Bestandteil der Manipulation, weil diese nicht immer mit Falschinformationen vonstatten gehen müssen. Auch ist Heimlichkeit kein notwendiges Kriterium für Manipulation: Wir kennen das, wenn die Eltern anrufen und fragen, "ob wir noch leben" oder "dass wir uns ja schon lange nicht mehr gemeldet haben«; was hier passiert, wissen wir meist genau: Es wird Schuld induziert, uns wird ein schlechtes Gewissen gemacht. Das geschieht ganz offen und transparent, ohne ernsthafte Verschleierung also. Zudem müssen die Konsequenzen der Manipulation nicht notwendigerweise negativ sein. Derselbe hier wirksame Mechanismus kann auch für eine Manipulation mit positiven Folgen genutzt werden. Wohl wahr ist aber, dass Manipulation unsere Rationalität zumindest teilweise unterläuft bzw. umgeht (wenn auch nicht komplett ausschaltet - sonst wären wir wieder im Zwangsbereich, in dem wir gar nicht mehr anders handeln könnten), indem sie auf unserer affektiven Ebene operiert.
} 
Zwang, verwischt werden. Manipulation kann als eigene Form der Beeinflussung verstanden werden, bei der ein Manipulator jemandem die Wahl eines Zweckes nahelegt, der Manipulierte aber frei in der Wahl bleibt, diesen Zweck zu seinem zu machen - oder nicht. Der Mechanismus, der hier im Spiel ist, besteht in der gezielten Verbindung einer Handlungsoption mit angenehmen oder unangenehmen Empfindungen. So werden manche Zwecke attraktiver oder unattraktiver und damit wahrscheinlicher gewählt oder nicht gewählt. Damit lässt sich Manipulation als Typus der Einflussnahme auf menschliches Handeln definieren, der von Täuschung, Zwang sowie rationalem Überzeugen unterschieden werden kann. So ist Manipulation eine attraktive Form der Beeinflussung nicht zuletzt in modernen, liberalen Gesellschaften, da den Manipulierten weiter die Entscheidungsfreiheit bleibt, während mittels der affektiven Ebene beeinflusst wird. Manipulierte Akteurinnen werden in Entscheidungssituationen noch immer durch ihre eigenen Affekte bewegt, die durch ein Aussen - also einen Manipulator oder eine extra dafür geschaffene Entscheidungsarchitektur ${ }^{25}$ - moduliert sein mögen, aber nicht künstlich erschaffen wurden. Der Einbezug von Affekten mag dabei eine Entscheidung für oder gegen etwas erschweren, weil nicht primär die Rationalität unsere Entscheidung bestimmt. Generell aber wird durch die Beeinflussung jenseits unseres rationalen Radars die freie Entscheidung für oder gegen etwas nicht unmöglich gemacht, wenngleich sie mitunter herausfordernder werden mag. Wir haben es mit einem Stupsen und manchmal gar Schubsen ins Affektive zu tun. Ein Handlungszwang in eine bestimmte Richtung allerdings entsteht daraus nicht, der manipulierten Akteurin bleibt die Wahl. Beschreiben wir die Manipulation auf diese Weise, indem wir ihre Wirkweise in den Fokus nehmen, haben wir ein neutrales Verständnis von Manipulation gewonnen, das Raum sowohl für negative als auch positive Formen lässt. ${ }^{26}$ Der manipulative Umgang

\footnotetext{
${ }^{25}$ Vgl. zum Thema Entscheidungsarchitekturen das immens erfolgreiche Buch von Richard Thaler/Cass Sunstein, Nudge. Improvising Decisions About Health, Wealth and Happiness, London 2009.

${ }^{26}$ Einen alltagssprachlich positiven Begriff gibt es für die emotionale Beeinflussung nicht. "Emotionale Beeinflussung" wäre vielleicht ein neutraler Kandidat, der weniger pejorativ konnotiert ist als "Manipulation" (was mit dessen Begriffsgeschichte v.a. nach der Zeit des Nationalsozialismus und dem aufstrebenden Kapitalismus im 20. Jahrhundert, der unsere Begierden ins Unendliche treibt, zu tun hat). Die negative Konnotation hat allerdings mehr mit einer Abneigung gegen eine Herausforderung der Rationalität zu tun als mit dem eigentlichen Wortsinn von "Manipulation" (was wiederum gerade vor dem Hintergrund der Propaganda des Dritten Reiches, dem aufstrebenden Populismus in der Welt zu unserer Zeit und der sinnlosen Stimulation
} 
mit Angst scheint ethisch betrachtet prima facie zur dunklen Seite der Manipulation zu gehören - doch selbst hier ist es nicht so einfach. Dazu an abschliessender Stelle mehr. Versuchen wir zunächst noch kurz zu sortieren, wie Angst manipulativ eingesetzt werden kann.

Es hat den Anschein, dass unterschiedliche Affekte unterschiedliche Wirkungen bei unterschiedlichen Personen in unterschiedlichen Kontexten entfalten können. Angst kann letztlich so vielfältig eingesetzt werden, wie sie sich auch zu äussern vermag. Versuchen wir hierfür die Kontexte für Angsterleben zu unterteilen: Für die Manipulation, die immer mindestens zwei Personen in Interaktion benötigt, ist vor allem der Bereich des Miteinanders relevant. ${ }^{27}$ Blicken wir also zunächst auf Beziehungen. Angst tritt hier - das ist einer der offensichtlichen Fälle - (1) im Rahmen psychischer Bedrohungen auf. Mit einer Zielsetzung, das Handeln der zu manipulierenden Person zu ändern, werden relativ schlichte Formen emotionaler Gewalt, in Form mehr oder minder konkreter Bedrohung (kommunikativ, aber auch mit Objekten) eingesetzt. Das geschieht, wenn man einschüchtert, also z.B. in ruhigstem Ton angedeutet wird, dass man den anderen zu verletzen gedenkt, wenn er $\mathrm{X}$ nicht tut. Die Unberechenbarkeit solcher schwebender (also ohne zwingende Gefahr des Eintretens seienden) Drohungen, überhaupt unberechenbares Handeln, fördert die manipulative Wirkung noch, indem das phantasievolle Kopfkino anspringt. Auch das Taschentuch, das William Shakespeares Jago in Othello in den Händen des Konkurrenten deplatziert, damit Othello (der eigentliche Schenker des Gegenstands) denkt, Desdemona betrüge ihn, verkörpert eine akute, aber auch ein Stück weit diffuse (denn man kann sich nicht ganz sicher sein) Bedrohung, dass nämlich Othello schon von der Bettkante gestossen und seine Liebe verloren ist. Manipulativ ist die

von Begierden im Kapitalismus nachvollziehbar ist). Da wir allerdings alle begrenzt rationale Wesen sind (siehe hierzu exemplarisch Herbert A. Simons Forschung), müssen wir vielleicht aber eingestehen, dass die Manipulation ein ganz normaler Modus der Kommunikation ist. Darum belasse ich es bei dem Begriff "Manipulation", der für eine Beeinflussung, die generell jenseits des rationalen Radars verfahrt, traditionell genutzt wird. Ich versuche aber zu differenzieren, das Deskriptive (also was eigentlich bei der Manipulation genau vonstatten geht) und das Normative (wie wir das, was dort vonstatten geht, bewerten sollten) zu trennen und zu fragen, worauf es eigentlich ankommt, wenn die Manipulation ins moralisch verwerfliche abrutscht und wie sie moralisch legitim sein kann. Mehr hierzu in: Fischer, Manipulation, 26ff. 117ff. 202ff. ${ }^{27}$ Ich lasse hier solche Ängste, die durch körperliche Erkrankung entstehen (z.B. die Angst zu ersticken, sich nicht mehr bewegen zu können o.̈.) oder spezifische Raumängste wie die Agoraphobie oder Klaustrophobie aussen vor, wenngleich es natürlich denkbar ist, eine Entscheidungsarchitektur für einen Klaustrophoben zu gestalten, die zu einer bestimmten Handlung führen soll. 
emotional-gewalttätige Androhung von Versehrung oder jedweden unberechenbaren, angedeuteten negativen Konsequenzen gerade deshalb, weil sie die "kreative Selbstqual« des manipulierten Akteurs stimuliert und eine bestimmte Handlungsweise als (von der Manipulatorin) präferierte markiert. ${ }^{28}$ Die Handlungsweise also sich einfach genau so zu verhalten, wie es die Manipulatorin möchte, mag selbst dem Manipulierten dann mitunter als kleineres Übel, weniger energieraubend oder ungefährlicher und deswegen zumindest (in einem weiten Sinne verstanden) angenehmer erscheinen, als sich zur Wehr zu setzen. Indem hier Energie aus der kognitiv-rationalen Deliberation auf das phantastische Ausmalen umgeleitet und von der kritischen Reflexion, der Möglichkeit eines Realitätsabgleichs, weggezogen wird, werden wir in die unangenehme Empfindung der Angst geschubst. Die Suche nach (guten) Gründen für unser Handeln und damit auch unser Handlungsspielraum sind so kontaminiert von Angst, weshalb das denkerische Ergebnis dann meist weit $\mathrm{ab}$ von sonst rational ermittelten, guten Gründen liegt. Dies geschieht auch durch die eintretende Zermürbung und damit konkrete Schwächung einer beständigen Drohatmosphäre, die unsere rationalen Kapazitäten schwächt und unserWohlfühlen unterminiert. Dadurch werden wir im schlimmsten Falle beständig manipulierbar und unfähig zur eigenen Befreiung.

Ein weiteres, komplexeres Szenario in der zwischenmenschlichen Interaktion, das sich Angst manipulativ zunutze macht, ist (2) das Spiel mit Anerkennung. Natürlich geht auch sie grundsätzlich mit Bedrohung einher (wie es bei Angst eben ist), geht dabei aber oft geschickter und subtiler vor. Im Spiel der Anerkennung zeigt sich unsere anthropologische Angewiesenheit auf andere, auf deren Schutz, Geborgenheit und Wertschätzung. Die Bedrohung und damit die Quellen der Angst bestehen in Zurückweisung, Liebesentzug, Trennung, Einsamkeit und damit auch genereller $\mathrm{Ab}$ - und Entwertung. Anders gesagt: Es besteht eine grundsätzliche Angst vor Bestrafung und verweigerter Anerkennung davon, in Ordnung zu sein, wie man ist. Oder nochmal anders gesagt: Die negative Bewertung von Aussen ist es, die uns mitunter schlottern lässt. Solche Angst mag sich mit ihren Folgen körperlicher Art sowie des gehemmten Fluchtimpulses, der umgehend einsetzenden Trennung von der Aussenwelt (mitunter Dissoziation) und ausgemalten Horrorszenarien,

${ }^{28}$ Konkrete körperliche Gewalt kann im grösseren manipulativen Kontext eingesetzt werden, um langfristig einzuschüchtern und ein bestimmtes Handeln zu generieren, ist aber in der einzelnen konkreten körperlichen Auseinandersetzung selbst nicht als Form der Manipulation zu verstehen. 
dass man beispielsweise auf ewig allein bleiben wird, zeigen. Dieses existenzielle Fundament der Angst bildet einen Wachstumsgrund für drei mitunter besonders toxische Verwandte, die ein teuflisches Wechselspiel mit der Angst initiieren: Scham, Schuld und auch Eifersucht (die jeweils unterschiedliche Anbindung an Angst haben). In toxischen Beziehungen wird manipulativ eine Stimmung des Nicht-Genügens kreiert und genutzt, um eine der Manipulatorin zuträgliche Handlungsoption (wenngleich möglicherweise mit dem Erbringen eines "Opfers « verbunden) als die attraktivere erscheinen zu lassen, verbunden mit der Hoffnung auf Seite des Manipulierten, dass dadurch Zuneigung, Liebe - Anerkennung (für das Bemühen z.B.) - ausgelöst wird.

Scham, als der unangenehme emotionale Zustand, in dem wir spüren, dass wir Massstäben nicht genügen, von denen wir denken, dass unser Gegenüber sie anlegt und dabei deutlich sieht, dass wir sie nicht erfüllen, wir aber glauben, sie erfüllen zu müssen, ist in direkter Wechselwirkung mit Angst zu verstehen: Wir genügen nicht, also könnte das schlimme Folgen haben (abgesehen davon, dass wir eh und je an den internalisierten Massstäben gescheitert sind, was sich schon generell nicht gut anfühlt). Die Angst steigert sich, was wiederum die Scham steigern kann. Schuld gilt Freud als »topische Abart der Angst ${ }^{29}{ }^{29}$ Sie wird in den ersten Lebensjahren als soziales Mittel erlernt und ist so in unserer kleinkindlichen "Hilflosigkeit und Abhängigkeit von anderen leicht zu entdecken, kann am besten als Angst vor dem Liebesverlust bezeichnet werden. $\aleph^{30}$ Schuld können wir also als den Glauben verstehen, etwas nicht richtig gemacht zu haben, wobei man jemandem womöglich gar etwas angetan hat (und darum wiederum nicht genügt - hier zeigt sich die enge Verbindung zur Scham). Sie bringt dabei die Angst vor der Strafe mit sich, also einer negativen Bewertung, einer konkreten Sanktion und in der Folge wiederum des Entzugs der Anerkennung. Hier zeigt sich, wie Konkretes und Diffuses in der Angst durcheinander gehen können. In der Eifersucht stehen die Dinge noch etwas anders. Eifersucht scheint eine toxische Gemengelage aus verschiedenen Emotionen zu sein - hierbei prominent auch die Angst. Wir müssen hier bereits mit dem akuten Schmerz der verloren geglaubten Anerkennung und dem ganz konkreten Verlust eines Liebesobjektes umgehen. Es zeigt sich also einerseits ein starker Bezug auf ein spezifisches Objekt im Aussen, während Scham und Schuld stark uns Selbst zum Objekt haben. So ist Eifersucht einerseits vielleicht ähnlich dem von Tieren

\footnotetext{
${ }^{29}$ Freud, Das Unbehagen in der Kultur, 495.

${ }^{30}$ Ebd., 483.
} 
bekannten Zubeissen aus Angst, da sie sich oft aggressiv nach Aussen richtet. Andererseits ist sie aber auch eine besonders kreativ-selbstquälerische Emotion (was die grosse nach Aussen gerichtete Energie füttert), denn sie lässt uns nicht nur schreckliche Szenen des Betrugs ausmalen, sondern ängstigt uns auch ganz existenziell, indem wir auch hier Horrorszenarien von z.B. ewiger Einsamkeit imaginieren. Eifersucht, wie Angst, lähmt unseren Neokortex, den anatomisch jüngsten Teil des Gehirns, der für das Rationale, die Sprache, das Logische zuständig ist. Sie tut dies derart, dass alle Zwischenrufe des Denkens in den Wogen der negativen Empfindung untergehen, die durch unser für das Empfinden primär zuständige limbische System ausgelöst wird. Der Einsatz scham-, schuld- oder eifersuchtsinduzierender Mittel ist im Rahmen der angstbezogenen Manipulation daher besonders wirksam, da sie in uns mit Leichtigkeit die Angst hervorrufen, nicht in Ordnung zu sein, keine Anerkennung mehr $\mathrm{zu}$ bekommen und somit verstossen zu werden. Das ist besonders unangenehm und birgt die Gefahr, gefügig die von der Manipulatorin bevorzugte Handlungsoption zu wählen. Auch, wenn dies nicht zwangsläufig die Folge sein muss, aber dafür braucht es oft viel Kraft, Distanz und am besten eigentlich die volle Funktionsfähigkeit und Handlungsleitung der rationalen Reflexion.

Die Frage der Anerkennung spielt auch in politischen Kontexten eine grosse Rolle, in denen Angst als manipulatives Mittel genutzt wird. Verschiedene Zeiten haben verschiedene Ängste als politische Faktoren genommen. Während es vor einigen Jahrzehnten etwa noch die Angst vor der Atomkraft oder Genmais gab, haben wir es in unserer jetzigen Zeit eher mit Angst vor den Folgen der Klimakrise (für jene, die sie leugnen, wie auch jene, die sie anerkennen), der Angst vor "Überfremdung" (die im weiteren Sinne eine Angst vor Non-Konformismus ist) und der Angst vor sozialem Abstieg, zu tun. Allen drei genannten modernen Ängsten (ohne Anspruch auf Vollständigkeit) liegt die Frage der Anerkennung zugrunde, wenn es um Position und Privilegien in der Gesellschaft geht. Der Soziologe Niklas Luhmann meint, dass sich in der durchfunktionalisierten Gesellschaft vor allem die Angst mit ihrer zeitlosen Anpassungsfähigkeit als eine Art Supersystem etabliert hat, das in der schwer bis unmöglich zu besorgenden Widerlegbarkeit von Angstgründen dazu führt, dass in der Bevölkerung eine Heuristik entsteht im Stile von: "Was Angst mindert, ist gut". Die Angst wird dabei von politischen Akteuren gezielt geschürt (typischerweise unter Betonung des Schlimmen und unter Auslassung des Guten, Funktionalen), bei gleichzeitiger 
Positionierung als Retter. ${ }^{31}$ Es wird dabei ein immerwährendes »Antizipieren des Schreckens" praktiziert und so eine win gewisser Weise [gewollte] Katastrophe" heraufbeschworen, die niemals vollständig widerlegbar ist und so Angst bedingen kann. ${ }^{32}$ Und tatsächlich macht Angst in ihrer Wirkung als starker Affekt gefügig, da sie uns von unserer Rationalität abzutrennen vermag. Auch sogenannte Verschwörungstheorien mögen in diese Gemengelage gehören.

Luhmann beschreibt so einen wesentlichen Reflex des Populismus, der es darauf anlegt, mit diffusen Ängsten zu spielen, indem dieser Gefahren heraufbeschwört, die mehr oder meist weniger gegeben sind. ${ }^{33}$ Dabei gehört dieser Reflex zu den eigentlichen Grundbewegungen von Politik, die der Beseitigung von Ängsten verpflichtet ist. Das markiert schon ihren Anfang in Form der Vergesellschaftung zum gemeinsamen Vermindern der Unsicherheit. Angst ist also ein alltägliches Mittel politischer Kommunikation und dabei ein zwiespältiges Phänomen: Sie kann im positiven Sinne einerseits Solidarität befördern (wie es in der Anfangsphase der Corona-Krise 2020 gewesen sein mag) und wird andererseits vielfach im negativen Sinne geschürt und instrumentalisiert, um die politischen Zielsetzungen von politischen Akteuren zu erreichen. Die AfD macht uns das in Deutschland mit ihrer Instrumentalisierung von Gewaltverbrechen und des Themas Einwanderung zur Induktion von Angst deutlich. Auch in der Schweiz wird die Angst gezielt genutzt, wenn es um das Minarettverbot oder auch hier die Masseneinwanderung geht. Genauso wie Donald Trump den Freihandel als toxisch, Flüchtende als Terroristen und die Politik als korrupten Sumpf oder die Brexiteers in Grossbritannien die EU als Blutsauger darstellen. Angst wird in diesen Zusammenhängen zu Slogans umgewandelt und damit manipulativ breitenwirksam handhabbar. Die Slogans lassen sich direkt an unsere Fähigkeit zur Phantasie andocken, indem z.B. die Nutzung von Stereotypen in diffusen Andeutungen angstvolle Bilder entstehen lässt (was auch die starken Wahlergebnisse dort erklärt, wo es keine Migranten gibt). Diese werden dann wiederum mit Handlungsoptionen verbunden: Wählt uns, denn wir vermindern die Angst. Und wir erinnern uns: Was Angst vermindert, ist gut. Der wieder erstarkte Populismus scheint zu unserer Zeit in vielen

\footnotetext{
${ }^{31}$ Vgl. Niklas Luhmann, Ökologische Kommunikation. Kann die moderne Gesellschaft sich auf ökologische Gefährdungen einstellen?, Wiesbaden 2004, 237ff.

${ }^{32}$ Theodor W. Adorno, Aspekte des neuen Rechtsradikalismus. Ein Vortrag, Berlin 2019, 19. Für Adorno gehört die ständig an den Horizont gemalte Katastrophe als Grundlage für politisch nutzbare Angst zu einem wesentlichen Merkmal der öffentlichen Kommunikation rechtsradikaler Akteure.

${ }^{33}$ Siehe hierzu ausführlicher Fischer, Ein Parasit im Kokon des Schmetterlings?, 24ff.
} 
Ländern die stärkere Überzeugungskraft zu besitzen. Die induzierte diffuse Angst ist dabei ein vielgenutztes Mittel, um jemanden zu einer Handlung zu bewegen. Das Beseitigen der Ängste ist für die lockende Seite allerdings eher zweitrangig, denn so würde ja das Mittel zur Bewegung verloren gehen. Im politischen Kontext kann Angst ausserdem dafür eingesetzt werden, die Menschen zu frustrieren, so dass sie beispielsweise nicht wählen gehen, da z.B. alle Wahlausgänge negative Folgen zu haben scheinen. Das ist gerade bei unentschiedenen Wählern interessant (und wird vielfach über die Sozialen Medien besorgt). Es gibt schliesslich mehrere Wahlsysteme auf der Welt, die von nicht abgegebenen Stimmen profitieren - man denke nur an die U.S.-Wahl 2016, bei der Donald Trump mit nur wenigen Stimmen Vorsprung gewann und das womöglich, weil ein Teil der Wähler frustriert gar nicht erst wählen ging.

\section{Kurzer Ausblick zur Ethik der angstinduzierenden Manipulation}

Wenn wir uns so vergegenwärtigen, in welchen Bereichen Angst wie eingesetzt werden kann, sind wir bereits mitten drin in der Frage nach der moralischen Legitimität.Versuchen wir abschliessend diese noch kurz zu thematisieren. ${ }^{34}$ Wir haben es bei Angst mit einem schillernden Affekt im Rahmen der manipulativen Beeinflussung zu tun, die in manchen Zusammenhängen als absolut verwerflich gilt, in anderen wiederum als legitim, wenn sie ihre Funktion als gerechtfertigter Schutzmechanismus (in aller Kürze) bedient - man denke nur an die leichte Angstinduktion bei Kindern, die sich vor Fremden zumindest ein bisschen ängstigen sollen, damit sie vorsichtig bleiben.

Ihr Einsatz liesse sich als verwerflich markieren, vor allem dann, wenn eine Täuschung über das tatsächliche Ausmass einer Bedrohung involviert ist, sie egoistischen Zielsetzungen eines Manipulators dient und letztlich negative Konsequenzen für die Manipulierte zeitigen wird. Diese mögen vor allem auch darin bestehen, dass Angst - gerade mittels unserer Phantasie - unberechenbare Effekte haben kann und so zu einem schwer kontrollierbaren Selbstläufer werden kann. Dass politische Systeme, die Angst routiniert als Mittel nutzen, wenig Gutes hervorbringen, ist in der Geschichte und auch unserer Gegenwart beobachtbar. Hier zeigt sich auch (im Grossen

${ }^{34}$ Vgl. hierzu ausführlicher Fischer, Manipulation, 159ff. 
der Politik wie im Kleinen der zwischenmenschlichen Beziehungen), dass es kein verlässliches Mass gibt, dass sicherstellen könnte, dass es bei "leichter Angst", die manipulativ induziert werden soll, bleibt. Angst vermag es, die elementarste Grundlage von Beziehung, Vertrauen, mitunter zu veröden. Sie ist so als Mittel der Manipulation selbst bei wohlwollenden Absichten problematisch. ${ }^{35}$ Das sonst oft so Präzise der Manipulation ist hier kaum zu besorgen.

Wenn Angst manipulativ eingesetzt wird, bedarf es einer Beziehung zweier gut Vertrauter, die trägt, die es ermöglicht, die Angst gemeinsam zu halten. Das mag in therapeutischen Kontexten der Fall sein, wo die vorsichtige gezielte Induktion von Angst z.B. im Kontext von Traumaarbeit hilfreich sein kann. Dass uns die Art und Weise der manipulativen Beeinflussung via des Affektes Angst als illegitim erscheint, wenn sie mit Heftigkeit vorgeht (wobei diese sich oft selbst entkräftet, indem sie Reaktanz bei den zu Manipulierenden hervorruft) oder flächendeckend bzw. von grosser Dauer ist, braucht da kaum noch erwähnt zu werden. Hier sind die körperlichen, emotionalen und kognitiven Folgen verheerend. George Orwells 1984 führt uns diesen Schrecken einer auf Angst aufgebauten, umfassenden Entscheidungsarchitektur eindrücklich vor. Die ständige Bedrohung kulminiert in einem Satz: „DER GROSSE BRUDER SIEHT DICH ${ }^{36}$ (überlege also genau, wie du handelst), womit das Angstregime moderner Diktaturen (oder auch hierfür Vorbild seiender strafender Religionen) auf den Punkt gebracht ist. Hier werden unsere psychische Ökologie, also unsere Selbstwahrnehmung und die sinnvolle Erzählung unseres eigenen (und das der anderen) Handelns in Bezug auf die Umwelt und unsere Möglichkeit zum freiheitlichen Handeln in zweifelhaft starker Weise vernebelt. Schliesslich, so lässt sich vielleicht sagen, ist es vor allem unsere Sicht der Dinge in der Welt, die uns zu beruhigen vermag. Selten sind es die Dinge selber.

So mag Angst als Mittel der Manipulation im Gegensatz zu positiven Affekten mit ihrer negativen Empfindungsebene, der Gefahr

\footnotetext{
${ }^{35}$ Es ist also einerseits naheliegend gewesen, weswegen Hans Jonas zur Abwendung der ökologischen Krise eine "Heuristik der Furcht" ins Spiel brachte, da ebenjene Angst ein grosser Beweger im Politischen sein mag. Andererseits ist aber auch verständlich, weswegen er damit auf Kritik stiess, da Angst ein unwägbares Mittel im (hier) Politischen darstellt (vgl. Hans Jonas, Das Prinzip Verantwortung:Versuch einer Ethik für die technologische Zivilisation, Frankfurt a.M. 1979).Vittorio Hösle schlägt im Gegenzug bspw. vor, die Liebe der Menschen zu allem Lebendigen zu entfachen (vgl. Vittorio Hösle, Philosophie der ökologischen Krise. Moskauer Vorträge, München 1991).

${ }^{36}$ George Orwell, 1984, übersetzt v. Michael Walter, München 2000, 8.
} 
des Abgleitens in phantasierte Horrorszenarien, der Abtrennung des eigenen Selbst von der Aussenwelt und dem starken verhinderten Impuls der Flucht eine primär problematische Form der Manipulation bedingen. Und das hat Konsequenzen, nicht nur für unsere zwischenmenschlichen Beziehungen, in der Angst eigentlich keine Rolle spielen sollte. Das gilt auch für die Gestaltung der Politik, die zwar Unsicherheit beseitigen, sie aber niemals leichtfertig und gewohnheitsmässig als Mittel nutzen sollte. Gerade der Umgang populistischer Bewegungen mit den Ängsten der Menschen ist geradezu zynisch. Eine Politik allerdings, die gänzlich ohne diesen mächtigen Beweger auskommt (wenngleich es grosse Unterschiede geben mag), ist erst noch zu ermitteln.

- Dr. phil. Alexander Fischer lehrt und forscht als wissenschaftlicher Assistent am Philosophischen Seminar der Universität Basel. Zu seinen Themen zählen Manipulation, Emotionen, Ethik und Erzählen. Neben der wissenschaftlichen Arbeit ist er psychotherapeutisch und beraterisch tätig. 